\title{
ANGIO-RM DAS ARTÉRIAS CARÓTIDAS E VERTEBRAIS: ANÁLISE DE DIFERENTES TÉCNICAS DE VOLUME E DILUIÇÃO DE CONTRASTE EM APARELHO DE 1,0 T E GRADIENTE DE $15 \mathrm{mT} / \mathrm{m}^{*}$
}

\author{
Luiz Guilherme C. Hartmann', Angela M. Borri Wolosker', Giuseppe D'Ippolito ${ }^{1}$, Maria Lúcia Borri ${ }^{1}$
}

Resumo OBJETIVO: Avaliar as artérias cervicais (carótidas e vertebrais) por meio da angio-RM, utilizando-se diferentes dosagens e diluições de contraste paramagnético. MATERIAIS E MÉTODOS: Estudo prospectivo em 15 pacientes, com análise de 30 artérias carótidas comuns, 30 artérias carótidas internas, 30 artérias carótidas externas e $\mathbf{3 0}$ artérias vertebrais, utilizando-se diferentes volumes e dosagens de contraste paramagnético: grupo I - dose única (14 ml de Gd-DTPA); grupo II - dose dupla (28 $\mathrm{ml}$ de Gd-DTPA); grupo III - dose única e diluída a $50 \%$. A injeção de contraste foi realizada com a utilização de bomba injetora e com velocidades de injeção de $2 \mathrm{ml} / \mathrm{s}$ (grupo I) e $3 \mathrm{ml} / \mathrm{s}$ (grupos II e III). Os segmentos arteriais foram analisados por três examinadores em consenso de forma subjetiva, avaliando-se o grau de visibilidade, intensidade de contrastação e definição de seus contornos. RESULTADOS: Em todos os itens analisados os pacientes do grupo II apresentaram melhor resultado (visibilização total, boa contrastação dos vasos e contornos bem definidos). No grupo I houve boa visibilização das artérias carótidas e vertebrais, porém a intensidade de contraste e a definição dos contornos apresentaram variabilidade qualitativa. $\mathbf{O}$ grupo III apresentou os piores resultados, com dificuldade de visibilização, intensidade de contrastação baixa e contornos mal definidos. CONCLUSÃO: Entre os grupos analisados, a técnica utilizando dose dupla de gadolínio é a que permite melhor avaliação das artérias cervicais. A utilização de contraste diluído prejudica a avaliação dos vasos do pescoço.

Unitermos: Angio-RM. Gadolínio. Artérias carótidas.

Abstract Contrast-enhanced MR angiography of the carotid and vertebral arteries: analysis of different contrast volumes and dilution techniques at $1.0 \mathrm{~T}$ with $15 \mathrm{mT} / \mathrm{m}$ gradient.

PURPOSE: To evaluate the carotid and vertebral arteries by magnetic resonance angiography, using different contrast volumes and dilution techniques. SUBJECTS AND METHODS: Prospective study of 15 patients, which included the evaluation of $\mathbf{3 0}$ common carotid arteries, 30 internal carotid arteries, 30 external carotid arteries and 30 vertebral arteries, using different gadolinium (Gd-DTPA) volumes and dilutions: group I - single-dose (14 $\mathrm{ml}$ of Gd-DTPA); group II - double-dose ( $28 \mathrm{ml}$ of Gd-DTPA); group III - single-dose, dilution at $50 \%$. GdDTPA was administered using a power injector at $2 \mathrm{ml} / \mathrm{sec}$ (group I) and $3 \mathrm{ml} / \mathrm{sec}$ (groups II and III). A qualitative assessment was performed for each arterial segment. Three radiologists, who judged the quality of depiction of the arteries, vascular signal intensity and contours delineation, performed a consensus analysis. RESULTS: All evaluated features showed better performance in group II (complete depiction of the arteries, good vascular signal intensity and well defined contours). In group I, there was adequate depiction of the arteries, but inadequate vascular signal intensity and contours delineation. Group III showed the poorest results, with inadequate parameters for all evaluated features. CONCLUSION: Among the tested Gd-DTPA dilutions and volumes, double-dose contrast injection allowed better evaluation of the carotid and vertebral arteries. The studies with dilution of Gd-DTPA showed the poorest results.

Key words: MR angiography. Gadolinium (Gd-DTPA). Carotid arteries.

\section{INTRODUÇÃO}

A doença aterosclerótica envolvendo a bifurcação carotídea, associada a eventos tromboembólicos, corresponde a uma causa importante de acidentes vascula-

* Trabalho realizado no Serviço de Ressonância Magnética do Complexo Hospitalar São Luiz, São Paulo, SP.

1. Médicos Radiologistas do Setor de Tomografia Computadorizada/Ressonância Magnética do Hospital São Luiz.

Endereço para correspondência: Prof. Dr. Giuseppe D'Ippolito. Hospital São Luiz, Centro Diagnóstico. Rua Dr. Alceu de Campos Rodrigues, $143,1^{\circ}$ subsolo. São Paulo, SP, 04544-000. E-mail: scopo@brhs.com.br

Aceito para publicação em 10/4/2001. res isquêmicos. Evidências observadas em estudos randomizados prospectivos, incluindo o North American Symptomatic Carotid Endarterectomy Trial (NASCET) $)^{(1,2)}$, o European Carotid Surgical Trial $^{(3)}$ e o Department of Veterans Affairs Symptomatic Carotid Stenosis Trial ${ }^{(4)}$, têm demonstrado redução do risco relativo de acidentes isquêmicos de $70 \%$ a 85\% quando a endarterectomia é realizada em pacientes com estenose igual ou acima de $70 \%$. Recentemente, os resultados finais do NASCET mostraram que pacientes selecionados com estenoses de até $50 \%$ se beneficiam de tratamento cirúrgico ${ }^{(5)}$. A indicação cirúrgica depende de uma série de fatores, entre eles a precisa determinação do grau de estenose das artérias carótidas.

A angiografia digital cerebral é até hoje o exame padrão-ouro para a avaliação pré-operatória. Nos últimos anos têm sido desenvolvidas novas técnicas de imagem não-invasivas para selecionar candidatos cirúrgicos, entre as quais citamos o ultra-som com Doppler (US Doppler) colorido $^{(6,7)}$, a angiografia por ressonância magnética (angio-RM) sem contraste 
(técnicas 2D e 3D TOF e "phase-contrast") ${ }^{(\mathbf{8}, \mathbf{9})}$ e a angiografia por tomografia computadorizada (angio-TC) ${ }^{(\mathbf{1 0 , 1 1 )}}$.

Essas técnicas, apesar de terem utilidade diagnóstica, apresentam limitações, seja por causa do restrito campo de visão (US Doppler), pelos artefatos e longo tempo de aquisição (angio-RM sem contraste) ou, ainda, pela exposição à radiação e trabalhoso processamento da imagem (angio-TC) ${ }^{(\mathbf{8 , 1 0})}$.

A introdução de estudos de angioRM com contraste paramagnético (gadolínio $)^{(\mathbf{1 2})}$ e o aparecimento de sequiências com curto tempo de aquisição permitiram o estudo do território carotídeo com melhor qualidade do que com a angioRM convencional, minimizando os artefatos que são frequientes nas sequiências 2D e 3D $\mathrm{TOF}^{(12)}$, sendo já considerado, por alguns autores, como método correspondente à angiografia digital e com resultados coincidentes ${ }^{(13-15)}$.

Contudo, para garantir a qualidade do exame de angio-RM com gadolínio, é necessário otimizar o tempo de aquisição e a administração do meio de contraste em relação ao volume, concentração e velocidade de injeção ${ }^{(16,17)}$.

O tempo de aquisição é importante, porque a seqüência deve ser adquirida na primeira passagem do meio de contraste nas artérias carótidas para evitar a contrastação de estruturas venosas, as quais, quando presentes, prejudicam a análise arterial. Este problema foi solucionado após a introdução de técnicas para cálculo do tempo ideal de aquisição e do uso de bombas injetoras ${ }^{(\mathbf{1 8 - 2 0 )}}$.

Porém, em relação à administração ideal do meio de contraste, isto é, o volume, a concentração (diluição) e a velocidade de injeção do meio de contraste, ainda não há consenso na literatura e tampouco trabalhos comparando diferentes diluições e volumes.

Trabalhos recentemente publicados têm descrito técnicas avançadas, utilizando, principalmente, seqüências em tempo real - denominadas, por alguns, de seqüências fluoroscópicas ${ }^{(21-23)}$-, que minimizam esses problemas. Essas técnicas necessitam, no entanto, de aparelhos com gradientes potentes e eficientes e programas específicos de alto custo, res- tritos a alguns centros médicos de grande porte.

Consideramos importante, assim, a tentativa de otimizar a angio-RM com gadolínio das artérias carótidas e vertebrais realizadas com aparelho de 1,0 T e gradiente de $15 \mathrm{mT} / \mathrm{m}$, considerados "standard" do equipamento na época da sua aquisição.

\section{Objetivo}

O objetivo deste trabalho foi avaliar a qualidade de visibilização das artérias cervicais (artérias carótidas comuns e internas e artérias vertebrais) por meio da angio-RM, utilizando-se diferentes dosagens e diluições do contraste paramagnético em um aparelho de 1,0 T operando com gradiente de $15 \mathrm{mT} / \mathrm{m}$.

\section{MATERIAIS E MÉTODOS}

\section{Técnica de exame}

Utilizamos um aparelho Philips NT10, com campo magnético de 1,0 T, gradiente de $15 \mathrm{mT} / \mathrm{m}$ e "slew rate" de 17 $\mathrm{mT} / \mathrm{m} / \mathrm{s}$, com "rise time" de 0,9 ms. Para a aquisição angiográfica foi utilizada seqüência volumétrica (3D) gradiente-eco ponderada em T1 (TR: 7,4 ms; TE: 2,7 ms; NSA: 1; "flip angle": 50; matriz: $256 \times 256$; FOV: $300 \mathrm{~mm}$ ), utilizando-se bobina de corpo.

A seqüência consistia em um bloco de 40 cortes com $1 \mathrm{~mm}$ de espessura e $1 \mathrm{~mm}$ de intervalo, sendo obtidas três fases por estudo (uma pré-contraste e duas póscontraste), todas em apnéia, com tempo de duração de 14 segundos por fase. Após a aquisição das imagens, as segunda e terceira fases foram reconstruídas com técnica "maximum intensity projection” (MIP) após subtração da primeira fase (pré-contraste).

A primeira seqüência pós-contraste foi obtida cinco segundos após o início da administração intravenosa de gadolínio. Este tempo foi determinado baseando-se em estudos prévios com angio-RM com contraste das artérias carótidas, que utilizaram o conceito de que a primeira aquisição deve ser completada na pequena janela de tempo, variando entre cinco e oito segundos, entre o pico de contrastação arterial e a subida da curva de contrastação venosa ${ }^{(17)}$.
Foi considerada dose simples de contraste paramagnético aquela correspondente a $0,2 \mathrm{ml} / \mathrm{kg}(0,1 \mathrm{mmol} / \mathrm{kg})$ de peso em paciente pesando $70 \mathrm{~kg}$ ( $14 \mathrm{ml}$ de contraste), fixando-se este valor para todos os pacientes dos grupos I e III. A dose dupla de contraste foi convencionada em 0,4 $\mathrm{ml} / \mathrm{kg}$, ou seja, $28 \mathrm{ml}$ em todos os pacientes do grupo II.

Em todos os casos do presente estudo foi utilizado gadopentato de dimeglumina (Gd-DTPA), em concentração de 469 $\mathrm{mg} / \mathrm{ml}$ (Magnograf ${ }^{\circledR}$, Justesa Imagem), injetado por meio de bomba injetora (Spectris $^{\circledR}$, Medrad).

\section{Grupos estudados}

Analisamos, prospectivamente, 15 indivíduos (nove mulheres e seis homens) submetidos a RM de crânio com gadolínio, sendo excluídos os pacientes com sintomas relacionados a doenças específicas das artérias carótidas e vertebrais, além de pacientes com antecedentes isquêmicos ou traumáticos. A idade dos pacientes variou de 18 a 70 anos, com idade média de 40 anos.

As artérias carótidas comuns, carótidas internas e vertebrais, em um total de 90 artérias, foram estudadas com diferentes dosagens e diluições de contraste, divididos em três grupos de cinco indivíduos selecionados de forma randomizada (totalizando 30 artérias por grupos), denominados de grupos I, II e III.

No grupo I foi administrada dose única de contraste $(14 \mathrm{ml})$ injetada à velocidade de $2 \mathrm{ml} / \mathrm{s}$. No grupo II foi administrada dose dupla de contraste $(28 \mathrm{ml})$ injetada à velocidade de $3 \mathrm{ml} / \mathrm{s}$. No grupo III foi administrada dose única de contraste diluída a $50 \%$ em água destilada, totalizando $28 \mathrm{ml}$ de solução, injetada também à velocidade de $3 \mathrm{ml} / \mathrm{s}$.

\section{Avaliação das imagens}

A qualidade das imagens das artérias carótidas comuns e internas, incluindo as bifurcações carotídeas e das artérias vertebrais, foi analisada por três observadores em consenso, que classificaram de forma qualitativa (subjetiva) os seguintes critérios:

a) visibilização do vaso, classificando-a como ausente, parcial ou total; 
b) intensidade de contrastação do vaso, classificando-a como ruim, regular ou boa;

c) definição dos contornos do vaso, classificando-os como mal definidos ou bem definidos.

Após a classificação das imagens, foi realizado estudo comparativo simples entre os grupos, procurando-se estabelecer qual destes apresentava globalmente as melhores imagens. Os examinadores não tinham conhecimento da técnica utilizada quando interpretaram os exames de angio-RM.

\section{RESULTADOS}

Observamos que todos os segmentos arteriais estudados num mesmo paciente mostravam qualidade de imagem semelhante, ou seja, a qualidade da imagem da artéria carótida comum direita era semelhante à da artéria carótida comum esquerda e à das artérias vertebrais em um mesmo paciente. Isto permitiu que agrupássemos as seis artérias estudadas em cada paciente na análise dos resultados, descritos a seguir (Tabelas 1, 2 e 3 ).

Tabela 1 Resultados quanto à visibilização das artérias cervicais nos três grupos estuda$\operatorname{dos}(\mathrm{n}=30)$

\begin{tabular}{|l|c|c|}
\hline \multirow{2}{*}{ Grupos } & \multicolumn{2}{|c|}{ Visibilização } \\
\cline { 2 - 3 } & Total & Parcial \\
\hline I (14 ml) & 24 & 6 \\
II (28 ml) & 30 & 0 \\
III (14 + 14 ml) & 30 & 0 \\
\hline
\end{tabular}

O grupo I, que recebeu aproximadamente $14 \mathrm{ml}$ de contraste sem diluição, apresentou 80\% (24/30) de visibilização total e $20 \%$ (6/30) de visibilização parcial, contrastação ruim em $60 \%$ dos casos, regular em $20 \%$ e boa em $20 \%$, e contornos mal definidos em $80 \%$ dos casos e bem definidos em apenas $20 \%$.

O grupo II, que recebeu aproximadamente $28 \mathrm{ml}$ de contraste (dose dupla), apresentou visibilização total e contrastação boa em todos os casos, com contornos bem definidos em $80 \%$ dos casos.

O grupo III, que recebeu aproximadamente $14 \mathrm{ml}$ de gadolínio diluído a 50\%,
Tabela 2 Resultados quanto à contrastação das artérias cervicais nos três grupos estuda$\operatorname{dos}(n=30)$.

\begin{tabular}{|l|c|c|c|}
\hline \multirow{2}{*}{ Grupos } & \multicolumn{3}{|c|}{ Contrastação } \\
\cline { 2 - 4 } & Ruim & Regular & B oa \\
\hline I (14 ml) & 18 & 6 & 6 \\
II (28 ml) & 0 & 0 & 30 \\
III (14 + 14 ml) & 0 & 30 & 0 \\
\hline
\end{tabular}

totalizando $28 \mathrm{ml}$, teve visibilização total em todos os casos, contrastação regular em todos os casos e contornos mal definidos em todos os casos.

As Figuras 1 e 2 ilustram esses resultados, mostrando exemplos das imagens obtidas nos três grupos.

\section{DISCUSSÃO}

$\mathrm{O}$ advento da angio-RM com injeção de dose dupla de contraste, inicialmente descrita por Prince, em 1994(16), permitiu que o método ocupasse, progressivamente, o espaço da angiografia digital no diagnóstico de doenças vasculares, tendo sido demonstrados resultados superponíveis e eficácia semelhante entre os dois métodos, com indiscutíveis vantagens da angio-RM, devido principalmente à sua baixa invasividade e à elevada comodidade para o paciente ${ }^{(\mathbf{1 3}-15)}$.

O contraste paramagnético (gadolínio) tem como principal característica diagnóstica a capacidade de aumentar o sinal
Tabela 3 Resultados quanto à definição dos contornos das artérias cervicais nos três grupos estudados $(n=30)$.

\begin{tabular}{|l|c|c|}
\hline \multirow{2}{*}{ Grupos } & \multicolumn{2}{|c|}{ Contornos } \\
\cline { 2 - 3 } & Definidos & Indefinidos \\
\hline I (14 ml) & 6 & 24 \\
II (28 ml) & 24 & 6 \\
III (14 + 14 ml) & 0 & 30 \\
\hline
\end{tabular}

emitido pelo sangue intravascular, encurtando significativamente o seu tempo de relaxamento T1. Nos exames de RM do crânio e corpo, geralmente é suficiente uma dose de gadolínio de $0,1 \mathrm{mmol} / \mathrm{kg}$ de peso, equivalente a $0,2 \mathrm{ml} / \mathrm{kg}^{(16)}$.

Aumentando-se as doses injetadas de contraste, melhora-se a qualidade de visibilização vascular, principalmente até uma concentração de $0,3 \mathrm{mmol} / \mathrm{kg}$, em virtude do expressivo encurtamento do T1. A partir desta dose não se observa melhora estatisticamente significante na qualidade da imagem, pois não se obtém uma queda apreciável do T1 do sangue no vaso ${ }^{(17)}$.

Dessa forma, difundiu-se o uso de dose dupla de contraste paramagnético $(0,2$ $\mathrm{mmol} / \mathrm{kg}$ ou $0,4 \mathrm{ml} / \mathrm{kg}$ ) nos estudos de angio-RM do corpo, com resultados bastante satisfatórios ${ }^{(13-15)}$.

Por outro lado, os custos dos exames diagnósticos têm sido motivo freqüente e crescente de preocupação na nossa sociedade, como pode ser visto em recente
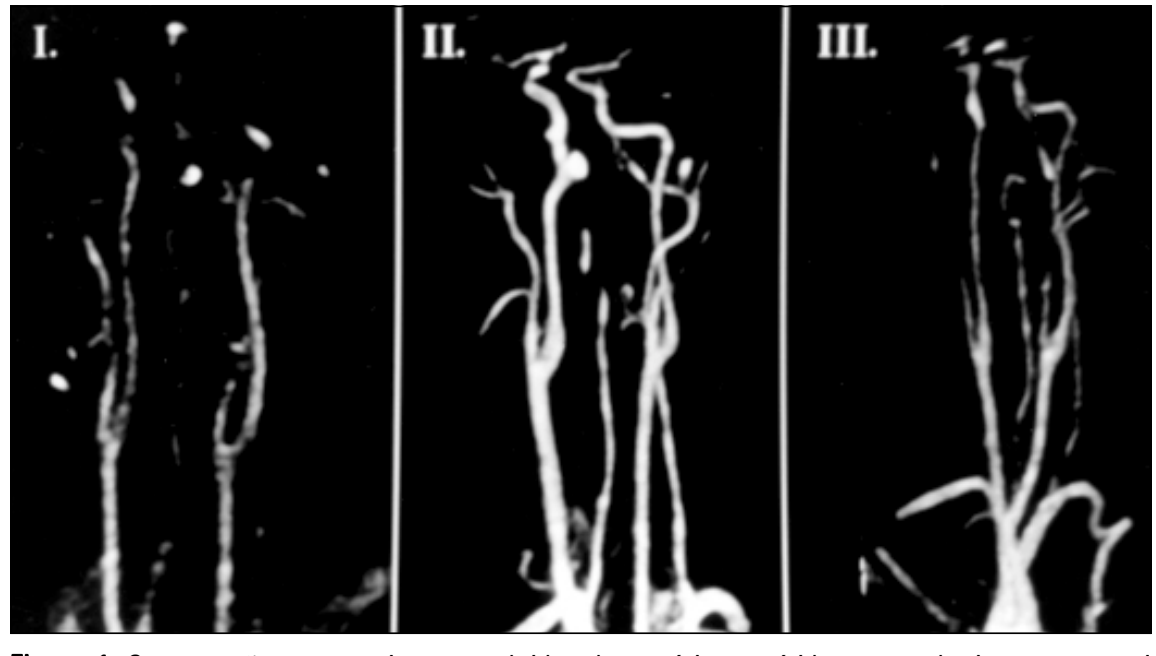

Figura 1. Comparação entre as imagens obtidas das artérias carótidas e vertebrais nos grupos (dose única de gadolínio), II (dose dupla de gadolínio) e III (dose única de gadolínio diluída a 50\%). 


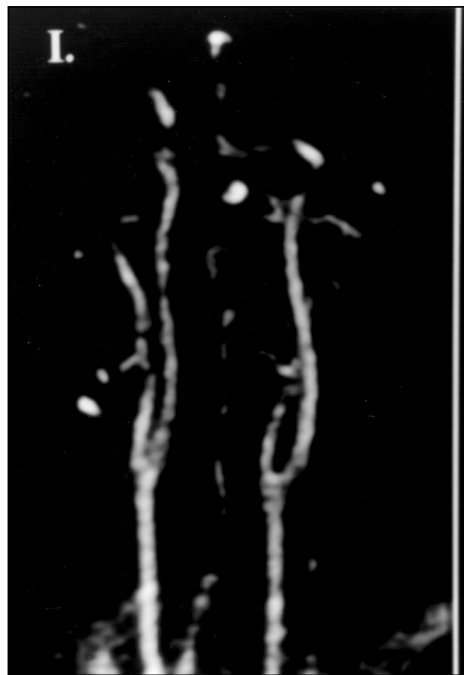

A

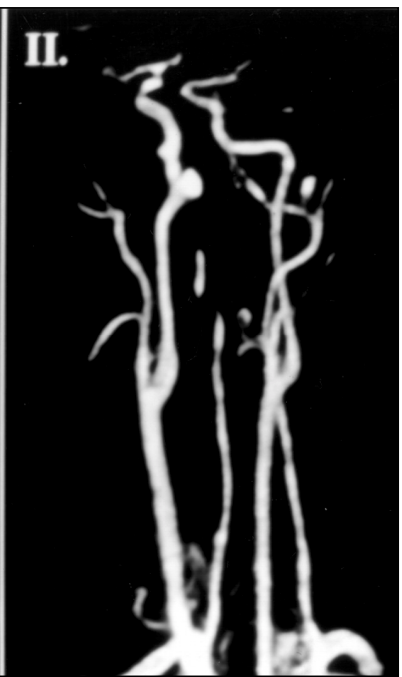

1

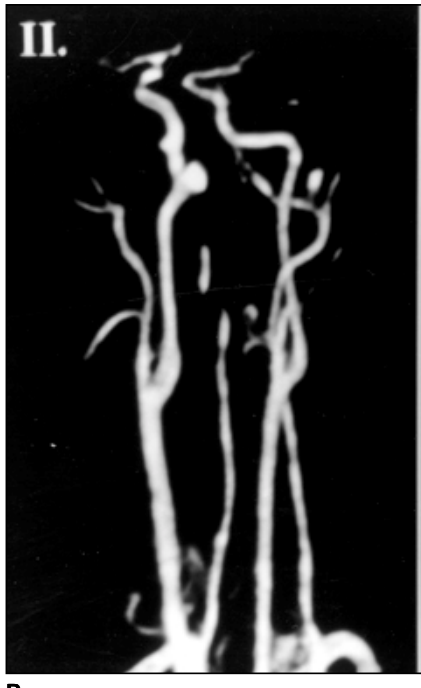

B

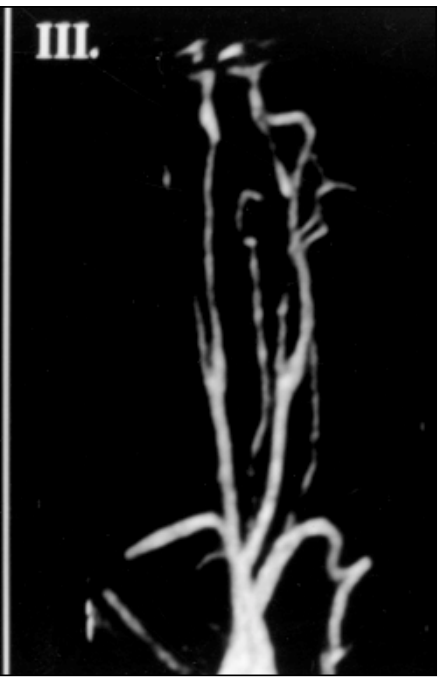

entre os grupos II e III (B). editorial no Radiology $\mathrm{y}^{(24)}$. O custo do contraste paramagnético tem influência importante no valor final do exame; portanto, a injeção de dose dupla ou tripla de gadolínio deve ser ponderada, procurando-se estabelecer a sua real necessidade e utilidade prática.

Recentemente, autores têm sugerido que o uso de dose simples de contraste paramagnético em estudos de angio-RM em magnetos operando em alto campo poderia ser suficiente para o diagnóstico preciso de doenças vasculares ${ }^{(25-27)}$.

Neste sentido, procuramos desenvolver este trabalho para estabelecer a real necessidade de dose dupla de gadolínio em estudos de angio-RM dos vasos cervicais, comparando os exames feitos com diversas doses e diluições.

Para isto, selecionamos pacientes sem doença vascular evidente e encaminhados para realizar exame de RM do crânio com contraste, pelas mais diversas indicações clínicas (por exemplo: estadiamento tumoral, suspeita de processo inflamatório, entre outras). Os pacientes foram sorteados aleatoriamente para serem examinados com um dos três protocolos, utilizando-se variadas doses e volumes de contraste. Optamos por não selecionar pacientes com suspeita de doença aterosclerótica carotídea, pois a nãovisibilização de um determinado vaso poderia ser atribuída à doença vascular e não à técnica utilizada.
Apesar de os melhores resultados em estudos de angio-RM cervical serem obtidos com o uso de bobina de quadratura ou de sinergia de pescoço, estas bobinas não foram utilizadas por causa de limitações técnicas, uma vez que o paciente estava sendo submetido à RM do crânio e, portanto, estudado com a bobina apropriada (bobina de quadratura de crânio), que não permite acoplar, concomitantemente, a bobina cervical. Por esta razão, todos os exames de angio-RM foram realizados com a bobina de corpo, que, como se sabe, apresenta limitada relação sinal/ruído, principalmente para pequenos campos de visão, como aqueles contemplados em estudos do pescoço.

Apesar dessa limitação técnica, todos os exames foram realizados da mesma forma, sendo esta desvantagem compartilhada entre os três grupos estudados. É possível que ao se usar bobina de sinergia ("phased-array") possa obter-se resultados diferentes daqueles por nós observados.

$\mathrm{O}$ número pequeno de pacientes examinados é outro limite do nosso estudo, que merece ser validado por casuísticas mais numerosas.

A maioria dos estudos com angio-RM do corpo recentemente publicados utilizam equipamentos operando em 1,5 T, com gradientes potentes $(23-30 \mathrm{mT} / \mathrm{m})$, tendo carência de resultados obtidos com magnetos operando em campo inter- mediário $(1,0 \mathrm{~T})$ e gradientes "standard" $(15 \mathrm{mT} / \mathrm{m})^{(28)}$, fato este que nos estimulou a realizar este trabalho.

A técnica de exame aqui empregada é aquela preconizada por outros autores, e excetuando-se o uso da bobina de corpo, pode ser considerada perfeitamente adequada, uma vez que utilizamos cortes finos (1 mm de espessura), técnica 3D, apnéia durante a infusão de contraste e pós-processamento com reconstruções tridimensionais com técnica MIP póssubtração ${ }^{(29)}$.

Outro parâmetro técnico que merece atenção é o intervalo de aquisição, ou seja, o início da aquisição das imagens em relação ao momento da injeção de contraste. Idealmente, deve-se determinar previamente o tempo de trânsito do contraste no vaso estudado, que pode variar em função da idade e do estado cardiocirculatório do paciente, e que pode ser calculado por diversas técnicas manuais ou automáticas ${ }^{(29)}$. Optamos por utilizar um tempo fixo de intervalo para o início da sequiência da angio-RM (cinco segundos a partir do início da injeção), considerando que na maioria dos pacientes as artérias carótidas iniciam-se a contrastar neste intervalo de tempo ${ }^{(23)}$, que a segunda seqüência obtida após a injeção de contraste seria capaz de recuperar aqueles exames em que o fluxo de contraste poderia ser mais lento, e por desconsiderarmos o efeito da contrastação das veias 
do pescoço sobre a qualidade final das imagens das artérias cervicais. Uma vez que nos três grupos estudados a técnica foi idêntica, acreditamos que o fator intervalo de aquisição não foi determinante nos resultados encontrados.

Este estudo foi baseado em avaliação meramente qualitativa (portanto, subjetiva). Posteriores estudos deverão ser realizados utilizando-se parâmetros quantitativos, como relação sinal/ruído e intensidade de sinal, entre outros, para validar os nossos resultados.

Os nossos resultados divergem dos de outros autores que compararam diversas doses de contraste em estudos de angioRM, como, por exemplo, o trabalho de Lentschig et al. ${ }^{(28)}$. Esses autores, ao estudarem a aorta toracoabdominal, observaram que não havia diferença estatisticamente significante ao se utilizar doses simples, dupla ou tripla de gadolínio. A discrepância de resultados pode ser decorrente do fato de termos analisado vasos de menor calibre e fluxo mais rápido, em que a influência da concentração e velocidade de injeção de contraste na qualidade da imagem é mais crítica.

A velocidade de injeção de contraste deve garantir que todo o volume infundido de gadolínio seja administrado até cinco a sete segundos antes do término da sequiência de aquisição das imagens $^{(28)}$. Pelo fato de a seqüência utilizada durar 14 segundos e o início da seqüência ocorrer cinco segundos após o início da injeção de contraste, procuramos selecionar uma velocidade de injeção que garantisse que o volume total de gadolínio fosse introduzido em até 12 segundos. Isto foi obtido utilizando-se uma velocidade de $3 \mathrm{ml} / \mathrm{s}$ para os grupos II e III (volume aproximado de $28 \mathrm{ml}$ ) e de $2 \mathrm{ml} / \mathrm{s}$ para o grupo I (volume aproximado de $14 \mathrm{ml}$ ).

Concluindo, observamos que empregando equipamento de RM operando a 1,0 T, com gradiente "standard", utilizando-se bomba injetora e bobina de corpo, os melhores resultados na contrastação dos vasos cervicais por meio de
angio-RM são obtidos utilizando-se dose dupla de contraste (aproximadamente igual a $28 \mathrm{ml}$ ). O uso de dose simples com ou sem diluição deve ser evitado, pois, apesar da indiscutível economia, os resultados são nitidamente inferiores.

\section{REFERÊNCIAS}

1. North American Symptomatic Carotid Endarterectomy Trial (NASCET). Methods, patient characteristics, and progress. Stroke 1991;22:711-20.

2. North American Symptomatic Carotid Endarterectomy Trial Collaborators. Beneficial effect of carotid endarterectomy in symptomatic patients with high-grade carotid stenosis. N Engl J Med 1991;325:445-53.

3. European Carotid Surgery Trialist's Collaborative Group. MRC European Carotid Surgery Trial: interim results for symptomatic patients with severe $(70-99 \%)$ or mild $(0-29 \%)$ carotid stenosis. Lancet 1991;337:1235-43.

4. Mayberg MR, Wilson SE, Yatsu F, et al. Carotid endarterectomy and prevention of cerebral ischemia in symptomatic carotid stenosis. Veterans Affairs Cooperative Studies Program 309 Trialist Group. JAMA 1991;266:3289-94.

5. Barnett HJM, Taylor DW, Eliasziw M, et al. Benefit of carotid endarterectomy in patients with symptomatic moderate or severe stenosis. North American Symptomatic Carotid Endarterectomy Trial Collaborators. N Engl J Med 1998;339: 1415-25.

6. Golledge J, Wright R, Pugh N, Lane IF. Colourcoded duplex assessment alone before carotid endarterectomy. Br J Surg 1996;83:1234-7.

7. Muto PM, Welch HJ, Mackey WC, O'Donnell TF. Evaluation of carotid artery stenosis: is duplex ultrasonography sufficient? J Vasc Surg 1996;24: $17-22$.

8. Patel MR, Kuntz KM, Klufas RA, et al. Preoperative assessment of the carotid bifurcation: can magnetic resonance angiography and duplex ultrasonography replace contrast arteriography? Stroke 1995;26:1753-8.

9. Huston J, Nichols DA, Luetmer PH, et al.MR angiographic and sonographic indications for endarterectomy. AJNR 1998;19:309-15.

10. Cumming MJ, Morrow IM. Carotid artery stenosis: a prospective comparison of $\mathrm{CT}$ angiography and conventional angiography. AJR 1994;163:517-23.

11. Dillon EH, van Leeuwen MS, Fernandez MA, Eikelboom BC, Mali WPTM. CT angiography: application to the evaluation of carotid artery stenosis. Radiology 1993;189:211-9.

12. Cloft HJ, Murphy KJ, Prince MR, Brunberg JA. 3D gadolinium-enhanced MR angiography of the carotid arteries. Magn Reson Imaging 1996;14: 593-600.

13. D'Ippolito G, Wolosker N, Galvão Filho M, Kalil JA, Wolosker A, Borri ML. Angio-RM com contraste no estudo da aorta tóraco-abdominal. Rev Imagem 1998;20:85-93.

14. D'Ippolito G, Wolosker N, Rosoky RMA, Galvão MM, Wolosker AMB, Borri ML. Rev Angiol 1999;15:117-20.
15. Shin JH, Suh DC, Choi CG, Lee HK. Vertebral artery dissection: spectrum of imaging findings with emphasis on angiography and correlation with clinical presentation. RadioGraphics 2000; 20:1687-96.

16. Prince MR. Gadolinium-enhanced MR aortography. Radiology 1994;191:155-64.

17. Prince MR, Grist TM, Debatin JF. 3D contrast MR angiography. 1st ed. Berlin: Springer-Verlag, 1997.

18. Slosman F, Stolpen AH, Lexa FJ, et al. Extracranial atherosclerotic carotid artery disease: evaluation of non-breath hold three-dimensional gadolinium-enhanced MR angiography. AJR 1998;170: 489-95.

19. Krinsky G, Maya M, Rofsky N, et al. Gadoliniumenhanced 3D MRA of the aortic arch vessels in the detection of atherosclerotic cerebrovascular occlusive disease. J Comput Assist Tomogr 1998;22: $167-78$.

20. Melhem ER, Caruthers SD, Faddoul SG, Tello R, Jara $\mathrm{H}$. Use of three-dimensional MR angiography for tracking a contrast bolus in the carotid artery. AJNR 1999;20:263-6.

21. Wilman AH, Riederer SJ, King BF, Debbins JP, Rossman PJ, Ehman RL. Fluoroscopically triggered contrast-enhanced three-dimensional MR angiography with elliptical centric view order: application to the renal arteries. Radiology 1997; 205:137-46.

22. Wilman AH, Riederer SJ, Huston J III, Wald JT, Debbins JP. Arterial phase carotid and vertebral artery imaging in 3D contrast-enhanced MR angiography by combining fluoroscopic triggering with and elliptical centric acquisition order. Magn Reson Med 1998;40:24-35.

23. Huston J III, Fain SB, Riederer SJ, Wilman AH, Bernstein MA, Busse RF. Carotid arteries: maximizing arterial to venous contrast in fluoroscopically triggered contrast-enhanced MR angiography with elliptic centric view ordering. Radiology 1999;211:265-73

24. Forman HP. Cost, value, and price: what is the difference and why care? Radiology $2001 ; 218$ : 25-6.

25. Korst MBJ, Joosten FB, Postma CT, Jager GJ, Krabbe JK, Barentsz JO. Accuracy of normal-dose contrast-enhanced MR angiography in assessing renal artery stenosis and accessory renal arteries. AJR 2000;174:629-34.

26. Ernst O, Asnar V, Sergent G, et al. Comparing contrast-enhanced breath-hold MR angiography and conventional angiography in the evaluation of mesenteric circulation. AJR 2000;174:433-9.

27. Tello R, Thomson KR, Witte D, Becker GJ, Tress BM. Standard dose Gd-DTPA dynamic MR of renal arteries. J Magn Reson Imaging 1998;8:4216

28. Lentschig MG, Reimer P, Rausch-Lentschig UL, Allkemper T, Oelerich M, Laub G. Breath-hold gadolinium-enhanced MR angiography of the major vessels at $1.0 \mathrm{~T}$ : dose-response findings and angiographic correlation. Radiology 1998;208: 353-7.

29. Watanabe Y, Dohke M, Okumura A, et al. Dynamic subtraction contrast-enhanced MR angiography: technique, clinical applications and pitfalls. RadioGraphics 2000;20:135-52. 